\title{
HIGHLIGHTS
}

LIVER

\section{Reduced mitochondrial dysfunction delays liver fibrosis}

New evidence indicates that hepatocyte mitochondrial dysfunction has a role in the early stages of liver fibrosis-a condition consistently associated with oxidative stress. Mitchell et al. induced fibrogenesis in transgenic mice that expressed the oncogene $B c l 2$ (encoding a membrane protein with mitochondrionspecific antioxidant properties) in their hepatocytes, because “...no studies had investigated the possible correlation between ... mitochondrial functional status and the different stages of fibrosis," explains Hélène Gilgenkrantz, the study's corresponding author.

The researchers injected both transgenic and wild-type mice with carbon tetrachloride, which induces liver fibrosis via increased formation of free radicals and reactive oxygen species (ROS). After either 3 or 6 weeks of carbon tetrachloride treatment, wild-type mice had significantly decreased respiratory chain complex activity and reduced mitochondrial DNA integrity compared with transgenic mice, which indicated a protective effect of $\mathrm{Bcl}-2$ with respect to mitochondrial dysfunction. Furthermore, following 3 weeks of carbon tetrachloride injections, liver tissue from transgenic mice contained $41 \%$ less fibrotic area (represented by sirius red staining in the accompanying figure) in comparison to tissues from wild-type mice. The decreased fibrosis was associated with reduced oxidative stress in the liver tissue from transgenic mice in comparison to wild-type mice. However, after 6 weeks of injections, the degree of fibrosis was comparable in the liver of both transgenic and wild-type mice.

The investigators suggest that Bcl-2-mediated protection against hepatocyte mitochondrial dysfunction delayed fibrosis but was not sufficient to prevent it, which they attribute to ongoing
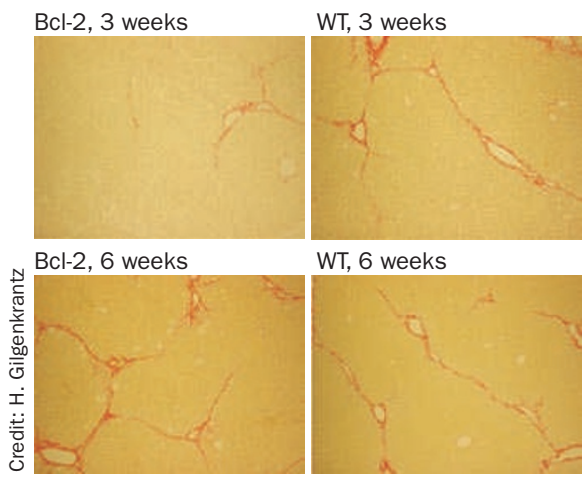

WT, 6 weeks

ROS generation in nonmitochondrial and/or nonhepatocytic components. Gilgenkrantz concludes by recommending that in disease conditions characterized by mitochondrial dysfunction, ROS generation and inflammation, combined treatments that target these features might slow the progression of fibrosis.

Rowan Higgs

Original article Mitchell, C. et al. Protection agains hepatocyte mitochondrial dysfunction delays fibrosis progression in mice. Am. J. Pathol. 175, 1929-1937 (2009) 